\title{
Avaliação da qualidade de óleos e/ou gorduras no processo de fritura em serviços de alimentação
}

Quality evaluation of oils and/or fats in the frying process in food services

Evaluación de la calidad de aceites y/o grases em el processo de fritura em servicios de alimentación

Recebido: 10/03/2021 | Revisado: 16/03/2021 |Aceito: 19/03/2021 | Publicado: 26/03/2021

\author{
Márcia Rejane Drescher \\ ORCID: https://orcid.org/0000-0002-6746-3961 \\ Universidade Franciscana, Brasil \\ E-mail: dreschermarcia@yahoo.com.br \\ Patrícia Arruda Scheffer \\ ORCID: https://orcid.org/0000-0002-2377-2930 \\ Universidade Franciscana, Brasil \\ E-mail: patyarruda9@gmail.com \\ Fernanda Dineia Viera \\ ORCID: https://orcid.org/0000-0003-3854-4807 \\ Universidade Franciscana \\ E-mail: fe_dineia@hotmail.com \\ Grazielle Castagna Cezimbra Weis \\ ORCID: https://orcid.org/0000-0003-0906-1224 \\ Universidade Federal de Santa Maria, Brasil \\ E-mail: grazielle.castagna@gmail.com \\ Cátia Regina Storck \\ ORCID: https://orcid.org/0000-0002-6491-6988 \\ Universidade Franciscana, Brasil \\ E-mail: catia.sm@gmail.com \\ Ana Lúcia de Freitas Saccol \\ ORCID: https://orcid.org/0000-0002-1253-8428 \\ Universidade Franciscana \\ E-mail: alsaccol@yahoo.com.br
}

\begin{abstract}
Resumo
Objetivo: avaliar a qualidade do óleo e/ou gordura utilizados no processo de fritura nos SA. Metodologia: Trata-se de um estudo de natureza descritiva, com abordagem quantitativa, fundamentado na avaliação do óleo de fritura em serviços de alimentação no município de Santa Maria/RS. Foram realizadas, no período de março a junho de 2019, visitas in loco, nos horários que ocorriam os procedimentos de fritura. Foi feito acompanhamento visual desse procedimento, aplicado um formulário para levantamento de informações e verificada a saturação do óleo por meio de fitas dosadoras. Os dados quantitativos foram tabulados em planilha Excel e analisados por meio de estatística descritiva simples. Resultados: Mais de $90 \%$ dos estabelecimentos realizam o processo de fritura diariamente. O óleo mais utilizado nesse processo é o vegetal (em 96,3\%). Em 74,1\% o óleo/e ou gordura era reposto quando este já estava reduzido, também em $74,1 \%$ o processo de fritura aconteceu de forma descontínua. Em 81,5\% não há controle da temperatura do óleo e/ou gordura durante o processo de fritura. Conclusão: Empregam de maneira incorreta o óleo e/ou gordura utilizados no processo de fritura. Isso pode acarretar diversos riscos à saúde. Destaca-se a importância da presença de profissionais da área de nutrição como ferramenta promotora de melhores práticas nesse processo, garantindo à população alimentos seguros e nutricionalmente adequados.
\end{abstract}

Palavras-chave: Inspeção de alimentos; Produção de alimentos; Refeições.

\begin{abstract}
Objective: to evaluate the quality of the oil and / or grease used in the frying process in SA. Methodology: This is a descriptive study, with a quantitative approach, based on the evaluation of frying oil in food services in the municipality of Santa Maria / RS. During the period from March to June 2019, on-site visits were carried out at the time of the frying procedures. A visual follow-up of this procedure was performed, a form was used to collect information and the saturation of the oil was verified through metering strips. The quantitative data were tabulated in Excel spreadsheet and analyzed using simple descriptive statistics. Results: More than $90 \%$ of the establishments conduct the daily frying process. The most used oil in this process is vegetable (96.3\%). In $74.1 \%$ the oil and/or fat was restored when it was already reduced, also in $74.1 \%$ the frying process happened in a discontinuous way. In $81.5 \%$ there is no control of the temperature of the oil and/or fat during the frying process. Conclusion: Incorrectly use the oil and/or fat used in the frying process. This can lead to various health risks. It is important to emphasize the
\end{abstract}


presence of nutrition professionals as a tool to promote best practices in this process, guaranteeing the population safe and nutritionally adequate food.

Keywords: Food inspection; Food production; Meals ready.

\section{Resumen}

Objetivo: evaluar la calidad del aceite y / o grasa utilizados en el proceso de fritura en SA. Metodología: Se trata de un estudio descriptivo, con enfoque cuantitativo, basado en la evaluación del aceite de fritura en los servicios de alimentación del municipio de Santa María/ RS. Durante el período de marzo a junio de 2019, se realizaron visitas in situ en el momento de los procedimientos de fritura. Se realizó un seguimiento visual de este procedimiento, se utilizó un formulario para recolectar información y se verificó la saturación del aceite mediante tiras dosificadoras. Los datos cuantitativos se tabularon en una hoja de cálculo de Excel y se analizaron mediante estadísticas descriptivas simples. Resultados: Más del $90 \%$ de los establecimientos realizan el proceso de fritura diaria. El aceite más utilizado en este proceso es el vegetal (96,3\%). En el 74,1\% se restauró el aceite y / o grasa cuando ya estaba reducido, también en el $74,1 \%$ el proceso de fritura se realizó de forma discontinua. En el 81,5\% no hay control de la temperatura del aceite y / o grasa durante el proceso de fritura. Conclusión: Utilizar incorrectamente el aceite y / o la grasa utilizados en el proceso de fritura. Esto puede ocasionar diversos riesgos para la salud. Es importante destacar la presencia de los profesionales de la nutrición como herramienta para promover las mejores prácticas en este proceso, garantizando a la población una alimentación segura y nutricionalmente adecuada.

Palabras clave: Inspección de alimentos; La producción de alimentos; Comidas listo.

\section{Introduçãa}

Ao longo dos anos, o comportamento alimentar passou por mudanças significativas, influenciadas, entre outros fatores, pela falta de tempo no dia a dia. Os participantes de sua pesquisa revelaram que consomem, com bastante frequência, comidas de restaurantes por causa da rapidez e praticidade com que são preparadas. (Viero \& Blumke, 2016).

No Brasil, por exemplo, de acordo com o estudo de Bezerra et al. (2013), o consumo de alimentos fora de domicílio foi reportado por $40 \%$ das pessoas. Índice similar ao dos Estados Unidos, onde pesquisa de Powell et al., (2012) revelou que $36 \%$ dos adultos consumem alimentos fora do lar.

Logo, "está havendo uma substituição da alimentação realizada em casa por restaurantes ou lanchonetes" (Viero \& Blumke, 2016), pois, nos dias de hoje, muitas pessoas vivem uma vida agitada e, por isso, cada vez mais elas optam por comer fora de casa. (Liu \& Tsai, 2010).

Assim, o crescimento geral dos serviços de alimentação (SA), segundo Saksena et al. (2018), é, em parte, uma resposta a essas transformações nos padrões de consumo. Para acompanhar esse crescimento, conforme Oliveira (2006), o mercado dos SA vem passando por mudanças significativas nas últimas décadas: o ingresso das redes de fast food no Brasil nos anos de 1970 e 1980 provocou uma reformulação de conceitos operacionais, a busca por informações técnicas mais apuradas e a implantação de sistemas de qualidade.

Diante desse cenário de aumento do consumo de refeições fora de domicílio, é fundamental que estabelecimentos que sirvam alimentos, como restaurantes, lanchonetes, etc., preocupem-se com a segurança dos alimentos que servem. Para Lourenço e Carvalho (2006), o gestor de um restaurante além de conhecer os procedimentos das Boas Práticas de Manipulação dos Alimentos (BPM), deve entender do processo produtivo das refeições, bem como proporcionar qualificações aos manipuladores de alimentos de sua equipe, tendo ciência de que a segurança alimentar está diretamente ligada à saúde pública, comprometendo-se com o atendimento das exigências da legislação vigente.

Segundo Osawa, Gonçalves e Mendes (2010), a expansão generalizada das redes de alimentação nos últimos anos tem aumentado consideravelmente o consumo de alimentos fritos. Jorge e Lopes (2003) confirmam que o processo de fritura tem sido amplamente empregado nos estabelecimentos comercias, como as redes de fast food, bares, lanchonetes, restaurantes, entre outros. 
De acordo com Martins, Broilo e Zani (2014), os óleos e/ou gorduras são usados na preparação dos alimentos para realçar o sabor, melhorar a textura e produzir o aroma desejado, sendo que a fritura é uma das formas de se utilizar os óleos na preparação de alimentos.

Sun et al. (2019) afirmam que alimentos fritos são altamente consumidos no mundo todo. Nesse sentido, para Cahill et al. (2014), a fritura é um método comum de cozinhar em diversos países. Pesquisa destes autores revelou que $14 \%$ das mulheres relataram consumir frituras entre 4 e 6 vezes por semana. Entre os homens, esse índice foi ainda maior: $23 \%$. No Brasil, os dados são ainda mais alarmantes, conforme o estudo de Zanini et al. (2013), 90\% dos jovens declararam consumir alimentos fritos pelo menos uma vez na semana; $28 \%$ destes consomem esse tipo de alimento todos os dias.

Esse alto consumo de frituras tem explicação similar ao do crescimento do consumo de alimentos fora de casa, de que o modelo de vida moderno, em que as pessoas têm menos tempo de preparar suas refeições, promove a utilização das frituras de modo excessivo, em virtude da opção de praticidade e de rapidez que a preparação desse tipo de alimento oferece. (Rabelo \& Ferreira, 2008; Batista et al., 2010).

Segundo Baptista e Linhares (2005), se as BPM não forem respeitadas pelo manipulador de alimentos, este pode ser responsável por colocar a saúde humana em risco. Os autores destacam a contaminação por substâncias químicas, formadas durante a preparação das refeições, que geralmente ocorre quando os alimentos são submetidos a processos em que a temperatura atingida no alimento é muito alta. A formação destes compostos químicos pode acontecer também pela prolongada exposição do alimento a uma temperatura adequada, mas que vai degradando o produto de forma progressiva. As gorduras e/ou óleos utilizados em processos de fritura são exemplos desta situação.

Silva, Araújo e Ferreira (2014) alertam para o fato de que óleos reutilizados de forma sucessiva perdem as características originais e se tornam ácidos, formando substâncias tóxicas como os radicais livres e ácidos graxos saturados. Esses, por sua vez, acarretam no envelhecimento precoce das células, irritação gástrica, além de doenças cardiovasculares e degenerativas.

Perante esse cenário, destaca-se a importância, segundo Rosa (2015), do uso correto das BPM de alimentos, que, para a autora, é uma maneira eficaz de diminuir o risco e melhorar o controle de qualidade para que os alimentos sejam oferecidos com maior segurança. Diante do exposto, o objetivo deste estudo é avaliar a qualidade do óleo e/ou gordura utilizados no processo de fritura nos SA.

\section{Metodologia}

Esta pesquisa trata-se de um estudo de natureza descritiva, com abordagem quantitativa (Prodanov \& Freitas, 2013; Soares et al, 2018). O trabalho foi fundamentado na avaliação da qualidade dos óleos e/ou gorduras utilizadas no processo de fritura em SA participantes do projeto de categorização no município de Santa Maria/RS.

Todos os estabelecimentos participantes do projeto foram convidados a participar desta pesquisa. Sendo realizada numa amostra de 27 estabelecimentos. Como critérios de inclusão, destacam-se a assinatura do termo de aceite e ter o processo de fritura por imersão como método de coç̧ão, e, como critério de exclusão, a ausência do processo de fritura nos SA.

As análises da qualidade do óleo e/ou gordura utilizado para fritura foram realizadas no período de março a junho de 2019, por meio de visitas in loco nos SA, nos horários que ocorriam os procedimentos de fritura. Foi realizado acompanhamento visual do procedimento de fritura, sendo também avaliados os aspectos sensoriais dos óleos e/ou gorduras. Foi feita a descrição do processo em diário de campo, e, posteriormente, aplicado um formulário para levantamento de informações quanto ao procedimento de fritura. O formulário foi aplicado na forma de entrevista com o responsável pelo procedimento. Aplicou-se um piloto do questionário em 5\% dos SA participantes, com intuito de testar os instrumentos da pesquisa. 
A temperatura do termostato do equipamento foi coletada, bem como foi verificada a saturação do óleo por meio de fitas dosadoras, da marca $3 \mathrm{M} \circledast$. Os dados foram registrados e tabulados em planilha excel e analisados por meio de estatística descritiva simples. Quanto aos aspectos éticos, esse projeto está vinculado à pesquisa intitulada "Implementação da Categorização dos Serviços de Alimentação em Santa Maria/RS”, do programa de pós-graduação em Ciências e Tecnologia de Alimentos da Universidade Federal de Santa Maria e possui aprovação no comitê de ética desta instituição, sob o número de parecer 2.890.701.

\section{Resultados e Discussão}

Dos 45 SA que assinaram o termo de adesão ao projeto de categorização no município de Santa Maria/RS, realizou-se a avaliação do processo de fritura em uma amostra representativa de $60 \%$ dos locais. Verificou-se que a maioria dos restaurantes utilizam a fritadeira elétrica com água embaixo para a realização do processo de fritura por imersão, e que, todas possuem termostato para controle da temperatura, conforme demonstrado na Tabela 1.

Por meio desse sistema, utiliza-se uma solução salina no fundo da fritadeira, onde ocorre a filtragem do óleo e/ou gordura, depositando ali os resíduos alimentares e aumentando assim a vida útil do óleo. Sendo assim, não há necessidade de filtrar o óleo e/ou gordura após o processo de fritura. Apenas dois estabelecimentos utilizam a frigideira nesse processo.

Tabela 1 - Característica do equipamento e óleo e/ou gordura utilizado no processo de fritura por imersão, Santa Maria (RS), 2019.

\begin{tabular}{ccc}
\hline Questões & Variáveis & $\mathbf{n}(\boldsymbol{\%})$ \\
\hline Onde é realizado o processo de fritura por imersão? & Fritadeira & $25(92,6)$ \\
\hline Qual a fonte de energia da fritadeira? & Frigideira & $2(7,4)$ \\
\hline Qual o tipo de fritadeira? & Elétrica & $24(88,9)$ \\
& A gás & $1(3,7)$ \\
\hline Oão usa Fritadeira & Com água embaixo & $13(5,4)$ \\
\hline processo de fritura? & Só óleo & $12(48,0)$ \\
\hline
\end{tabular}

Fonte: Construção das autoras.

De forma semelhante, no estudo de Martins, Broilo e Zani (2014), a maioria, 90,3\%, dos restaurantes pesquisados utilizava fritadeira elétrica, o que, segundo Mellema (2003), pode contribuir para reduzir a formação de Ácidos Graxos Trans (AGT) durante o processo de fritura. Isso porque o termostato possibilita a constante verificação da temperatura, que deve estar abaixo de $180^{\circ} \mathrm{C}$, evitando o superaquecimento do óleo. É importante destacar que a Anvisa orienta que o óleo deve ser filtrado a cada término de uso, aumentando assim a sua vida útil. (Brasil, 2004a).

$\mathrm{Na}$ tabela 2, tem-se que, entre os SA analisados, mais de $90 \%$ realizam esse processo diariamente, e os alimentos mais preparados são a batata frita, polenta frita e pastéis, respectivamente. Como já visto anteriormente, os alimentos fritos por 
imersão possuem uma ótima aceitação pela população em geral, principalmente nas refeições realizadas fora de casa, pela grande oferta, praticidade e rapidez. Além das características sensoriais que conferem, dão saciedade. Essa melhor aceitação se deve porque, segundo Pinto, Franco e Caldas (2016), esse tipo de fritura confere características específicas de odor, cor e sabor aos alimentos. Martins, Broilo e Zani (2014) também identificaram alto índice de frituras apresentadas no cardápio diariamente: em $87 \%$ dos estabelecimentos pesquisados. Em 8,1\% eram oferecidas no cardápio mais de 3 vezes na semana.

Tabela 2 - Periodicidade, tipo de óleo e/ou gordura, reposição e tipo de processo de fritura por imersão, Santa Maria (RS), 2019.

\begin{tabular}{ccc}
\hline Questões & Variáveis & n (\%) \\
\hline No serviço de alimentação, o processo de fritura é \\
realizado: & Diariamente & $25(92,6)$ \\
Qual é o óleo e/ou gordura utilizado? & 2 vezes na semana & $2(7,4)$ \\
\hline $\begin{array}{c}\text { Durante o processo de fritura é reposto o óleo e/ou } \\
\text { gordura quando este já estiver reduzido? }\end{array}$ & Óleo vegetal & $26(96,3)$ \\
\hline O processo de fritura acontece de forma contínua desde & Sim & $2(3,7)$ \\
\hline o início até a finalização? & Não & $7(74,1)$ \\
\hline
\end{tabular}

Fonte: Construção das autoras.

Quanto aos óleos, os mais utilizados são os vegetais, sendo predominantemente o de soja, e, em seguida, o de algodão (tabela 2). Um resultado positivo quanto ao aspecto nutricional, apenas um dos estabelecimentos empregava a gordura vegetal hidrogenada em seus processos de fritura. Valores similares aos encontrados na pesquisa de Borjes, Secon e Silva (2014), onde 93,3\% dos estabelecimentos relataram ser o óleo vegetal o mais utilizado no processo de fritura, seguido da gordura vegetal hidrogenada, $6,7 \%$.

Foi constatado ainda que, no decorrer do procedimento, os óleos e/ou gorduras são repostos à medida que estes vão reduzindo, bem como o procedimento, na maioria das vezes, dá-se de forma descontínua (Tabela 2). Sendo assim, a minoria dos estabelecimentos segue o que preconiza a Anvisa, onde deve-se dar preferência por fritar por longos períodos, ao contrário de utilizar a fritadeira/frigideira por vários períodos curtos, bem como alerta para o fato de que o ideal é não reutilizar o óleo de fritura, mas, caso haja real necessidade, deve-se evitar completar o óleo em uso com óleo novo. O ideal é descartar a sobra de um óleo, pois, ao completá-lo, a degradação do óleo adicionado será muito mais rápida. (Brasil, 2004a).

Nesse sentido, no estudo de Amaral et al. (2013), observou-se que a maioria (53,0\%) dos estabelecimentos também fazia reposição na fritadeira com óleo novo mais de uma vez por dia, 41,1\% faziam todos os dias. Apenas 5,9\% repõem o óleo nas fritadeiras com mais de 7 dias. Vários estudos, como o de Sanibal \& Mancini Filho (2004), Camilo (2007) e Bastos (2014) demonstram que as mudanças químicas que ocorrem no óleo durante o processo inadequado de fritura descontínua podem ser prejudiciais à saúde. 
No presente estudo, segundo dados apresentados na Tabela 3, a grande maioria dos restaurantes não controlam a temperatura do óleo e/ou gordura durante o processo de fritura e não apresentam planilhas de controle. Apenas seis estabelecimentos realizam esse controle por meio de termômetro, sendo a maioria do tipo laser $(83,3 \%)$.

Tabela 3 - Controle de temperatura do processo de fritura por imersão, Santa Maria (RS), 2019.

\begin{tabular}{|c|c|c|}
\hline Questões & Variáveis & n $(\%)$ \\
\hline \multirow{2}{*}{ Os manipuladores controlam a temperatura do óleo e/ou gordura? } & Sim & $5(18,5)$ \\
\hline & Não & $22(81,5)$ \\
\hline \multirow{3}{*}{ Se sim, como controlam? } & & $1(16,7)$ \\
\hline & Iermometro uро espeto & \\
\hline & Termômetro tipo laser & $5(83,3)$ \\
\hline \multirow{2}{*}{ Tem planilha de registro da temperatura? } & Sim & $5(18,5)$ \\
\hline & Não & $22(81,5)$ \\
\hline \multirow{2}{*}{ A temperatura no termostato para o procedimento de fritura é constante? } & Sim & $8(32,0)$ \\
\hline & Não & $17(68,0)$ \\
\hline
\end{tabular}

Fonte: Construção das autoras.

Declararam monitorar a temperatura durante o processo de fritura $87 \%$ dos restaurantes pesquisados no estudo de Martins, Broilo e Zani (2014). Esse controle da temperatura é importante, pois os óleos/gorduras sofrem alterações físicoquímicas ao serem submetidos a altas temperaturas.

Conforme demonstra-se na tabela 4, a maioria também não controla a temperatura fixada no termostato, favorecendo a oscilação e ultrapassando a temperatura recomendada. Já em relação a temperatura do óleo e/ou gordura verificados antes de adicionar o alimento, $68,3 \%$ dos estabelecimentos estavam de acordo com o recomendado, ou seja, abaixo de $180^{\circ} \mathrm{C}$. A temperatura média verificada foi de $161,1^{\circ} \mathrm{C}$.

Tabela 4 - Controle de temperatura do processo de fritura por imersão, Santa Maria (RS), 2019.

\begin{tabular}{|c|c|c|}
\hline Questões & $\mathbf{T}^{\circ} \mathbf{C}$ & n $(\%)$ \\
\hline \multirow{3}{*}{ Qual a temperatura máxima usada no termostato? } & $\leq 180$ & $5(20,0)$ \\
\hline & 200 & $8(32,0)$ \\
\hline & 300 & $12(48,0)$ \\
\hline \multirow{3}{*}{ Qual a temperatura fixada no termostato? } & $<180$ & $4(16,0)$ \\
\hline & 180 & $9(36,0)$ \\
\hline & $>180$ & $12(48,0)$ \\
\hline \multirow{5}{*}{ Temperatura do óleo ou gordura antes de adicionar o alimento: } & $\leq 140$ & $8(36,4)$ \\
\hline & 141 a 160 & $4(18,2)$ \\
\hline & $161^{\circ} \mathrm{C}$ a 180 & $3(13,7)$ \\
\hline & 181 a 200 & $5(22,7)$ \\
\hline & 201 a 220 & $2(9,0)$ \\
\hline
\end{tabular}


Nesse sentido, deve-se ter cuidado com esse processo, pesquisa de Mendonça et al. (2008) sobre as alterações físicoquímicas em óleos de soja submetidos a processo de fritura em unidades de produção de refeição demonstrou que óleos aquecidos a altas temperaturas, de forma repetida por um longo período de tempo podem passar por várias alterações físicas e químicas, produzindo compostos tóxicos prejudiciais à saúde humana. Dentre essas substâncias tóxicas (e até cancerígenas), estão a acroleína e peróxidos (Marques et al., 2009).

Jorge (2009) explica que essas alterações ocorrem porque a luz e o calor aceleram a decomposição das gorduras através da lipase, formando ácidos graxos livres (AGT’s), que são os causadores do sabor e odor desagradáveis. Para Freire et al., (2013) são os AGT’s os responsáveis por alterarem as características sensoriais do produto, diminuindo o ponto de fumaça do óleo/gordura de fritura. Assim, Pinto et al., (2016) destacam a importância de se conhecer as alterações sofridas pelos óleos durante o aquecimento para se otimizar os processos de fritura e, dessa forma, garantir produtos de melhor qualidade.

Por isso, a Anvisa determina que a temperatura máxima do óleo para fritura seja de $180^{\circ} \mathrm{C}$ (Brasil, 2004b, 2004a). Em âmbito estatual, a portaria 78/2009 da Secretária de Saúde do Rio Grande do Sul também estabelece que óleos e/ou gorduras aquecidos não devem ser submetidos à temperatura superior à $180^{\circ} \mathrm{C}$. Devendo-se monitorar a qualidade de óleos e gorduras para frituras com registros desse controle. (Rio Grande do Sul, 2009).

Tabela 5 - Armazenamento, troca do óleo e/ou gordura e higienização dos equipamentos utilizados no processo de fritura por imersão, Santa Maria (RS), 2019.

\begin{tabular}{ccc}
\hline Questões & Variáveis & $\mathbf{n}(\boldsymbol{\%})$ \\
\hline O óleo e/ou gordura é trocado: & Diariamente & $4(14,8)$ \\
& Semanalmente & $14(51,9)$ \\
& Quinzenalmente & $8(29,6)$ \\
As fritadeiras ou frigideiras são higienizadas: & Mensalmente & $1(3,7)$ \\
\hline O óleo e/ou gordura utilizados após o processo de & Diariamente & $6(22,2)$ \\
fritura permanece nas fritadeiras até o dia seguinte ou & Semanalmente & $13(48,1)$ \\
até o próximo procedimento? & Quinzenalmente & $7(25,9)$ \\
& Mensalmente & $1(3,7)$ \\
\hline O óleo e/ou gordura quando armazenados, são & Sim & $23(85,2)$ \\
guardados em recipientes fechados? & & $4(14,8)$ \\
& Não & $11(47,8)$ \\
\hline São armazenados em refrigeradores? & Sim & $12(52,2)$ \\
\hline
\end{tabular}

Fonte: Construção das autoras.

De acordo com a pesquisa, tem-se um resultado positivo em relação à troca do óleo e/ou gordura que é realizada na maioria dos SA semanalmente. Da mesma forma, ocorre a periodicidade com a higienização das fritadeiras e/ou frigideiras. Entretanto, o óleo e/ou gordura utilizados após o processo de frituras permanece nas fritadeiras até o dia seguinte ou até o 
próximo procedimento, e, quando armazenados, nem sempre são guardados em recipientes fechados e permanecem em temperatura ambiente.

No estudo de Martins et al., (2014) o tempo médio de utilização dos óleos e gorduras nas frituras também foi de até 15 dias, sendo que $43 \%$ dos estabelecimentos realizavam a troca a cada 7 dias. De forma semelhante, Borjes et al., (2014) avaliaram que, em grande parte (40\%) dos restaurantes, a frequência de descarte do óleo de fritura era quinzenal, em também $40 \%$ o descarte era feito semanalmente.

Flores-Alvarez et al., (2012) destacam que que os óleos reutilizados várias vezes para fritar acabam afetando a qualidade do alimento frito, justamente, segundo os autores, pelo fato de passarem por mudanças na composição química e nas características físicas. Quanto a higienização das fritadeiras, no Manual de Boas Práticas (MBP) da Anvisa (Brasil, 2004b), que é um documento que descreve as atividades executadas no estabelecimento e a maneira certa de fazê-las, há informações gerais sobre como a higienização deve ser feita, sobre treinamento dos manipuladores, como garantir a produção de alimentos seguros e saudáveis, dentre outras informações. Já o POP é um documento que detalhadamente descreve como executar as atividades no estabelecimento e que deve ser seguido de forma rigorosa para que tudo ocorra corretamente. Assim, o POP detalha as etapas da atividade, quem irá executá-la, quais os materiais necessários e a frequência em que deve ser feita.

Shinohara et al., (2016) afirmam que a inadequada manipulação, relacionada à higienização incorreta, é uma das principais fontes de contaminação dos alimentos. Dessa forma, consoante Saccol et al., (2006), as qualificações específicas para quem trabalha com alimentos são o meio mais recomendável e eficaz para transmitir conhecimentos e promover mudanças de atitude. Somente por meio de programas permanentes e eficazes, informação e conscientização é que será possível que se ofereça e produza alimentos seguros ao consumidor.

A Anvisa recomenda também que, em intervalos de uso, deve-se manter o óleo em recipientes tampados e abrigados da luz, evitando-se o contato com os principais catalisadores de oxidação, oxigênio e luz e, se o intervalo de uso for longo, deve ser guardado na geladeira. (Brasil, 2004a).

Foi constatado que a maioria não realiza teste para verificação da saturação do óleo e/ou gordura, conforme encontrase na Tabela 6. Dos que realizam, fazem através da percepção sensorial, e somente dois relataram usar a fita dosadora.

Tabela 6 - Controle de saturação do óleo e/ou gordura no processo de fritura por imersão, Santa Maria (RS), 2019.

\begin{tabular}{ccc}
\hline Questões & Variáveis & $\mathbf{n}(\mathbf{\%})$ \\
\hline $\begin{array}{c}\text { Realiza-se algum teste para o controle de saturação do óleo e/ou } \\
\text { gordura utilizados? }\end{array}$ & Sim & $6(22,2)$ \\
Se sim, como? & Fita de saturação & $21(77,8)$ \\
\hline & Avaliação sensorial & $2(28,6)$ \\
Verificar a saturação do óleo ou gordura antes de adicionar o & & $5(71,4)$ \\
alimento: & $2,0 \%$ & $2(8,7)$ \\
& $3,5 \%$ & $18(78,3)$ \\
\end{tabular}

Fonte: Construção das autoras.

Em relação a análise sensorial, de todos os estabelecimentos onde estavam ocorrendo o processo de fritura no momento, 34,8\% não apresentaram nenhuma alteração; 26,1\% apresentaram coloração escura; e também 26,1\% além da cor 
escura, apresentaram resíduos e 13,0\% apresentaram outras características indesejáveis (presença de fumaça, espuma e odor desagradável).

Já a verificação da saturação do óleo e/ou gordura foi realizada antes de adicionar o alimento em todos os estabelecimentos onde estavam realizando fritura naquele momento. Em 8,7\% dos estabelecimentos, o óleo estava com $2 \%$ de saturação, ou seja, o óleo e/ou gordura começou a degradar; em 78,3\% dos estabelecimentos o óleo estava com 3,5\% de saturação, devendo-se descartar o óleo e/ou gordura se a qualidade (cor, sabor, textura) dos alimentos fritos não for aceitável; em 4,3\% dos estabelecimentos, a saturação estava 5,5\%, devendo-se descartar o óleo e/ou gordura se a qualidade (cor, sabor, textura) dos alimentos empanados não for aceitável; e, em 8,7\% dos estabelecimentos, o óleo estava em 7,0\% de saturação, neste caso, recomenda-se o descarte do óleo e/ou gordura para todos os produtos alimentícios.

Já na pesquisa de Martins et al., (2014), a análise da saturação do óleo e/ou gordura em 82\% dos casos era sensorial (por sua cor e /ou odor), $8 \%$ fazia por meio de fita e 6,5\% não revelaram a forma de como realizavam o método de avaliação da saturação do óleo e/ou gordura. Para Mattos et al., (2000), o teste da fita indicadora é um método rápido, utilizado para obter resultados imediatos, servindo como um indicativo para monitorar a qualidade e o ponto de descarte dos óleos de fritura.

Os óleos e/ou gorduras devem ser substituídos quando houver evidência de alteração das características físicoquímicas ou sensoriais (fumaça, espuma, aroma e sabor indesejáveis) (Rio Grande do Sul, 2009). Por isso, Filipe (2013) afirma que avaliar a qualidade dos óleos de fritura é um importante fator para se obter produtos seguros e de qualidade.

Desta forma evidencia-se a necessidade de adequações nas BPM em serviços de alimentação, sendo assim uma estratégia para oferecer um alimento seguro para o consumidor (Melo et al., 2020). Para que ocorra o controle higiênicosanitário destes locais é necessário a presença de um responsável técnico (Souza et al., 2021).

Quanto a questão do descarte dos óleos e/ou gordura utilizados na preparação de alimentos fritos, devido a não existir uma regulamentação brasileira, somado ao fato da desinformação dos estabelecimentos de SA do país, é crucial a importância de dispor de métodos rápidos e simples que identifiquem o estado dos óleos e/ou gordura de fritura. (Amaral et al., 2013).

Sanibal e Mancini Filho (2004) comentam que não há somente um caminho para se definir o momento certo para se fazer o descarte de um óleo e/ou gordura utilizado na fritura, isto é, até quando é possível se utilizar um óleo e/ou gordura sem que o desempenho do produto seja comprometido negativamente e não traga prejuízos à saúde.

Dessa forma, as recomendações de BPM no processo de fritura devem ser seguidas para que se obtenha um melhor controle desse processo, garantindo uma melhor qualidade dos alimentos fritos (Ans et al., 1999). Além disso, Camilo (2007) chama a atenção para o fato de que urge, no Brasil, a instituição de legislação apropriada, abrangendo as variáveis e a qualidade do óleo durante o processo, bem como o manejo correto de descarte, objetivando estimular o seu reaproveitamento como técnica ecologicamente correta.

\section{Conclusão}

Os resultados desta pesquisa permitiram observar que, em relação ao processo de fritura por imersão, necessita-se de maior atenção por parte dos estabelecimentos comerciais. Empregam de maneira incorreta o óleo e/ou gordura utilizados nesse processo, utilizam temperaturas abusivas, bem como esse processo se dá de forma descontínua e fritam diversos tipos de alimentos numa mesma fritadeira, o que acelera a degradação. Outro fator preocupante, é o grande aumento do consumo de alimentos fritos diariamente.

Esse processo deve ser considerado uma questão relevante de vigilância sanitária no Brasil, já que não são adotados critérios físico-químicos confiáveis para determinar a qualidade dos óleos e/ou gorduras utilizados nesse processo. Desta forma, podem trazer implicações nutricionais à saúde, como: doenças crônicas não transmissíveis (DCNT), pré-disposição à aterosclerose, contribuindo para o risco de infarto do miocárdio, acidente vascular encefálico (AVE) e ação carcinogênica. 
Por conta disso, é de extrema importância atender às recomendações de boas práticas de fritura. Destaca-se também a importância de profissionais da área de nutrição como ferramenta promotora de melhores práticas nesse processo, garantindo à população alimentos seguros e nutricionalmente adequados.

Para futuras pesquisas, sugerimos que haja capacitações em boas práticas na manipulação do óleo, além de ações educativas com os manipuladores sobre o impacto da qualidade do óleo na saúde do consumidor. O treinamento continuo dos colaboradores é uma estratégia para a promoção de saúde, principalmente quando discutimos sobre alimentação coletiva.

\section{Referências}

Álvarez, M. D. C. F., Hernández, E. F. M., Raya, J. C. H., \& Morales, M. E. S. (2012). The Effect of Food Type (Fish Nuggets or French Fries) on Oil Blend Degradation during Repeated Frying. Journal of Food Science, 77(11), C1136-C1143. https://doi.org/10.1111/j.1750-3841.2012.02930.x

Amaral, D. A., Ferreira, V. F., Salvador, L. I. de S., \& Ferreira, C. C. (2013). Degradação de Óleos e Gorduras de Fritura de Pastelarias da Região Centro-Sul de Belo Horizonte, MG. HU Revista. https://periodicos.ufjf.br/index.php/hurevista/article/view/2038/755

Ans, V. G., Mattos, E. de S., \& Jorge, N. (1999). Avaliação da qualidade dos óleos de fritura usados em restaurantes, lanchonetes e similares. Ciência $e$ Tecnologia de Alimentos, 19(3), 413-419. https://doi.org/10.1590/s0101-20611999000300021

Baptista, P., \& Linhares, M. (2005). Higiene e Segurança Alimentar na Restauração. Forvisão.

Bastos, L. C. S., \& Pereira, P. A. de P. (2015). Estudo da formação de compostos carbonílicos, com ênfase em hidroxialdeídos $\alpha$, $\beta$-insaturados, na fase líquida de óleo de soja aquecido e avaliação da influência de íons metálicos. Universidade Federal da Bahia.

Batista, A. P. A., Fernandes, L. T. M., \& Lessa, N. M. V. (2010). Avaliação da quantidade de óleos e gorduras utilizados na preparação do cardápio semanal de uma unidade produtora de alimentos (UPA), o impacto à saúde do comensal e verificação do descarte adequado ao meio ambiente. Nutir Gerais, 624-636. https://silo.tips/download/ana-paula-arruda-batista-graduanda-em-nutriao-pelo-centro-universitario-do-leste

Bezerra, I. N., Souza, A. de M., Pereira, R. A., \& Sichieri, R. (2013). Consumption of foods away from home in Brazil. Revista de Saúde Pública, 47, 200211. https://doi.org/10.1590/S0034-89102013000700006

Borjes, L. C., Cecon, G., \& Silva, A. P. B. da. (2014). Análise da degradação do óleo de fritura de restaurantes comerciais do centro de chapecó-sc. DEMETRA: Alimentação, Nutrição \& Saúde, 9(3), 833-848. https://doi.org/10.12957/demetra.2014.8516

Brasil (2004 ). Ministério da Saúde. Agência Nacional de Vigilância Sanitária (ANVISA). Informe Técnico $n^{o} 11$ de 5 de outubro de 2004 . Óleos e gorduras utilizados em frituras.

Brasil (2004 ). Ministério da Saúde. Agência Nacional de Vigilância Sanitária (ANVISA). Cartilha sobre boas práticas para serviços de alimentação. (3a ed.).

Cahill, L. E., Pan, A., Chiuve, S. E., Sun, Q., Willett, W. C., Hu, F. B., \& Rimm, E. B. (2014). Fried-food consumption and risk of type 2 diabetes and coronary artery disease: A prospective study in 2 cohorts of US women and men. American Journal of Clinical Nutrition, 100(2), 667-675. https://doi.org/10.3945/ajen.114.084129

Camilo, V. M. A., \& Almeida, a D. T. (2007). avaliação do processamento de fritura por imersão em estabelecimentos comerciais da cidade do salvador Bahia [Universidade Federal da Bahia]. http://www.livrosgratis.com.br

Filipe, R. A. A. D. (2013). Óleos de fritura nas unidades de alimentação de instituição militar - comparação na aplicação de testes e implementação da boa prática no processo de preparo. Segurança Alimentar e Nutricional, 80-95. https://periodicos.sbu.unicamp.br/ojs/index.php/san/article/view/8634624/2545

Freire, P. C. M., Mancini-Filho, J., \& Ferreira, T. A. P. de C. (2013). Principais alterações físico-químicas em óleos e gorduras submetidos ao processo de fritura por imersão: Regulamentação e efeitos na saúde. Revista de Nutricao, 26(3), 353-358. https://doi.org/10.1590/S1415-52732013000300010

Jorge, N., \& Lopes, M. do R. V. (2003). Avaliação de óleos e gorduras de frituras coletados no comércio de São José do Rio Preto-SP. Alimentação e Nutrição, 14(2), 149-156. https://www.researchgate.net/publication/49600110_Avaliacao_de_oleos_e_gorduras_de_fritu ras_coletados_no_comercio_de_Sao _Jose_do_Rio_Preto-SP

Jorge, N. (2009). Química e Tecnologia de Óleos vegetais. Cultura Acadêmica.

Liu, C.-H., \& Tsai, W.-S. (2010). The effects of service quality and lifestyle on consumer choice of channel types: The health food industry as an example. African Journal of Business Management, 1023-1039. https://www.researchgate.net/publication/265451830_The_effects_of_service_qualit y_and_lifestyle_on_consumer_choice_of_channel_types_The_health_food_industry_as_an_example

Lourenço, M. S.; \& Carvalho, L. R. (2006). Segurança alimentar: utilização de ferramenta de qualidade para melhorias em um restaurante comercial. XIII SIMPEP. Bauru, São Paulo.

Marques, A. Y. C., Valente, T. B., \& da Rosa, C. S. (2009). Formação de toxinas durante o processamento de alimentos e as possíveis conseqüências para o organismo humano. Revista de Nutricao, 22(2), 283-293. https://doi.org/10.1590/S1415-52732009000200010

Martins, D. M. ., Broilo, M. ., \& Zani, V. . (2014). Óleos e gorduras: utilização no processo produtivo de restaurantes comerciais de Porto Alegre. Nutrire: Rev. Soc. Bras. Alimm. Nutr., 39(1), 25-39. https://doi.org/10.4322/nutrire.2014.004 
Mattos, E. S.; Ans, V. G.; \& Jorge, N. (2000). Utilização do Kit Oil Test para avaliação da alteração dos óleos de fritura. Higiene Alimentar, (11).

Mellema, M. (2003). Mechanism and reduction of fat uptake in deep-fat fried foods. Trends in Food Science and Technology, 14(9), 364-373. https://doi.org/10.1016/S0924-2244(03)00050-5

Melo, M. T. S. M., Santos, G. M. dos, Macêdo, M. C. F., Silva, B. L. dos S., Ripardo, S. R. P., Torres, B. L. M., Moura, A. C. C., Nunes, I. F. de O. C., Barreto, S. C. S., Paz, S. M. R. S. da, \& Carvalho, C. M. R. G. de. (2020). Segurança sanitária na produção de refeições em restaurantes de um mercado público no nordeste brasileiro. Research, Society and Development, 9(12), e2191210481. https://doi.org/10.33448/rsd-v9i12.10481

Mendonça, M. A., Borgo, L. A., Araújo, W. M. C., \& Novaes, M. R. C. G. (2008). Alterações físico-químicas em óleos de soja submetidos ao processo de fritura em unidades de produção de refeição no Distrito Federal. Com Ciências Saúde, 115-122.

Oliveira, M. S. (2006). Gestão de restaurante: uma prática de hospitalidade. Dissertação de Mestrado. Universidade Anhembi-Morumbi.

Osawa, C. C., Gonçalves, L. A. G., \& Mendes F. M. (2010). Avaliação dos óleos e gorduras de fritura de estabelecimentos comerciais da cidade de Campinas/ SP. As boas práticas de fritura estão sendo atendidas? Alimentos e Nutrição Araraquara, 21(1), 47-56.

Pinto, A. S. O., Franco, T. C. M., Souza, J. M., \& Caldas, R. N. A. (2016). Avaliação da qualidade de óleos de fritura no pre paro de alimentos comercializados em universidades de belém-pa. XXV Congresso Brasileiro de Ciência e Tecnologia de Alimentos.

Powell, L. M., Nguyen, B. T., \& Han, E. (2012). Energy intake from restaurants: Demographics and socioeconomics, 2003-2008. American Journal of Preventive Medicine, 43(5), 498-504. https://doi.org/10.1016/j.amepre.2012.07.041

Prodanov, C. C., \& Freitas, E. C. (2013). Metodologia do Trabalho científico: métodos e técnicas da pesquisa e do trabalho acadêmico. (2ed.). Universidade Feevale. www.feevale.br/editora

Rabelo, A. R.; \& Ferreira, M. O. (2008). Coleta seletiva de óleo residual de fritura para aproveitamento industrial. Universidade Católica de Góias, Goiana.

Rio Grande Do Sul (2009). Secretaria da Saúde. Portaria no 78 de 28 de janeiro de 2009. Aprova a Lista de Verificação em Boas Práticas para Serviços de Alimentação, aprova Normas para Cursos de Capacitação em Boas Práticas para Serviços de Alimentação e dá outras providências. Diário Oficial da União. Porto Alegre.

Rosa, P. T., \& Guelbe, T. F. (2015). Implantação do manual de Boas Práticas de Manipulação em cozinha pedagógica de uma instituição de ensino na cidade de campo mourão - PR. In Universidade Tecnológica Federal do Paraná- Departamento Acadêmico de Alimentos.

Saccol, A. L. de F., Rubim, B. de A., Mesquita, M. O., \& Welter, L. (2006). Importância de treinamento de manipuladores em boas práticas. Disc. Scientia. Série: Ciências Da Saúde. https://periodicos.ufn.edu.br/index.php/disciplinarumS/article/view/906/850

Saksena, M. J., et al. ( 2018). America's eating habits: food away from home. Economic Research Service. Disponvel em: < https://www.ers.usda.gov/publications/pub-details/?pubid=90227>

Sanibal, E. A. A., \& Mancini Filho, J. (2004). Perfil de ácidos graxos trans de óleo e gordura hidrogenada de soja no processo de fritura. Ciência e Tecnologia de Alimentos, 24(1), 27-31. https://doi.org/10.1590/s0101-20612004000100006

Shinohara, N. K. S., Almeida, A. Â. M. de, Nunes, C. G. P. da S., Lima, G. S. de, \& Padilha, M. do R. de F. (2016). Boas Práticas Em Serviços De Alimentação: não conformidade. Revista Diálogos Acadêmicos, 10, 79-91. http://www.semar.edu.br/revista

Silva, I. B. R. A, Araujo, R. A, \& Ferreira, N. A. (2014). Efeito da peroxidação de óleos utilizados em frituras na saúde humana effect of peroxidation used oil in frying on human health. In Faculdades Integradas ICESP/Promove de Brasília.

Soares, A., Dorlivete, P., Shitsuka, M., Parreira, F. J., \& Shitsuka, R. (2018). Metodologia da pesquisa cientifica .

Souza, A. O., Passos, E. V. B., Gonçalves, M. I. A., Coelho, R. L. L., \& Silva, C. de S. (2021). Perfil higiênico-sanitário de um restaurante comercial no interior baiano. Research, Society and Development, 10(2). https://rsdjournal.org/index.php/rsd/article/view/11870/11490

Sun, Y., Liu, B., Snetselaar, L. G., Robinson, J. G., Wallace, R. B., Peterson, L. L., \& Bao, W. (2019). Association of fried food consumption with all cause, cardiovascular, and cancer mortality: Prospective cohort study. BMJ (Online), 364. https://doi.org/10.1136/bmj.k5420

Viero, M. G., \& Blümke, A. C. (2016). A sociabilidade exercida em torno do comer: um estudo entre universitários. DEMETRA: Alimentação, Nutrição \& Saúde, 11(4). https://doi.org/10.12957/demetra.2016.15726

Zanini, R. de V., Muniz, L. C., Schneider, B. C., Tassitano, R. M., Feitosa, W. M. do N., \& González-Chica, D. A. (2013). Consumo diário de refrigerantes, doces e frituras em adolescentes do Nordeste brasileiro. Ciencia e Saude Coletiva, 18(12), 3739-3750. https://doi.org/10.1590/S1413-81232013001200030 